\title{
Überlieferung:
}

Aus Mensa i Valls, Arnau: guía bibliográfica, 67-76:

Bernkastel-Kues, Bibliothek des St. Nikolaus-Hospitals, 42, 15. Jh., fol. 210r217v (vgl. Perarnau 1981, 78-82 und Ed. Burnam, 146-148, 155).

Graz, UB, 1226, 14. Jh., fol. 21r-26r. Der Text ist abgekürzt (vgl. Ed. Burnam, 156-157).

Oxford, BodL, Ganon. misc. 370, 14. Jh., fol. 36v-53r (vgl. Ed. Burnam, 149-150, 157).

Saint-Omer, BM, 292, a. 1359, fol. 130v-139r. Der zweite Teil des Textes ist abgekürzt (vgl. Ed. Burnam, 150-152, 157-158).

Vaticano, BAV, Borgh. 205, 14. Jh. (Anfang), fol. 9r-20r (vgl. Maier 1948, 53-66 und Ed. Burnam, 141-145, 158).

Vaticano, BAV, Vat. lat. 3824, 14. Jh. (Anfang), fol. 29r-43r (vgl. Perarnau, 1992, 10-24 und Ed. Burnam, 135-141, 158-159).

Vaticano, BAV, Vat. lat. 5732, 15. Jh., fol. 83rb-89va (vgl. Ed. Burnam, 148-149, 159-160).

Hope L. Burnam beschreibt ferner in ihrer Edition, 146, 155-156:

Frankfurt a. M., StUB, Ms. Praed. 58, 14. Jh. (Ende), fol. 81r-86r.

Aus In principio:

Cambridge, UL, Mm. 6. 12, 15. Jh., fol. 31v-52. Vollständiger Text, Alcuinus zugeschrieben. Titel: Alcuini alphabetum catholicorum ad inclitum dominum regem Aragonum pro filiis erudiendis in elementis catholice fidei et est dyalogus.

Mensa i Valls weist auf eine Handschrift in Sydney hin, über deren Inhalt er nicht sicher ist. Der Bibliothekar Neil Boness hat für mich Incipit und Explicit überprüft. Nach seinen Angaben handelt es sich um das Alphabetum ohne die anschließende obieccio. Es könnte sich folglich um eine erste Fassung des Textes handeln (s. o. unter „Datierung und Fassungen"):

Sydney, Fisher Library of the University, Nicholson 23, 14./15. Jh., fol. 151r-163r. Unvollständig. Explicit: in unitate spiritus sancti Deus per omnia secula seculorum. Titel: Collocutio didascalica super elementis catholicae fidei magistri Arnaldi de Vilanova.

Hinzu kommt (Mitteilung von Josep Perarnau i Espelt):

Saint-Omer, BM, 361, 14. Jh. (2. H.), fol. 127r-134v. Abschrift der Hs. Saint-Omer, BM, 292, Text ebenfalls unvollständig.

\section{R37. Anonymus}

\section{Disputatio inter clericum et militem}

Rep.: RepFont 4, 210 und 8, 317; von Perger 95; Mohan, 296*.

Studie: Kapitel 3.4.2., 3.4.4.

Incipit: Primo proponit clericus miramentum quodammodo sub hac forma. Clericus: Miror, optime miles, paucis diebus tempora mutata... 
Explicit: ... sintque cum rege et intrante et egrediente. Unxit quoque foada pontifex [2 Par 6, 7, 11] ibi.

Autor: In der Handschrift Vat. Borgh. 29 werden Marsilius von Padua und Wilhelm von Ockham als mögliche Autoren angegeben. In den alten Drucken erscheint meistens Wilhelm von Ockham als Autor. Da der Text jedoch vor 1297 verfasst wurde, ist die Autorschaft des Marsilius (zwischen 1275 und 1280 geboren) aus chronologischen Gründen unwahrscheinlich, die Autorschaft Wilhelms (zwischen 1290 und 1300 geboren) unmöglich. Erickson argumentiert gegen deren Autorschaft mit stilistischen und inhaltlichen Argumenten (Ed. Erickson, 288). Scholz kommt in seiner Studie dieses Traktats zu folgenden Ergebnissen bezüglich des Autors: Wegen seiner Einstellung gegenüber dem Papst und der flandrischen Partei muss er Franzose sein; seine nicht allzutiefen Kenntnisse der Bibel und seine wörtliche Auslegung, auch sein Standpunkt in der Diskussion passen zu einem Laien; seine Argumentation zeigt, dass er eine scholastische und juristische Bildung hat (Scholz, 336-337). Scholz schlussfolgert „... dass er ein königlicher Legist, ein in der Politik des Hofes bewanderter Mann war..." (345). Miethke betrachtet eine kollektive Autorschaft als möglich, was die Anonymität erklären würde (De potestate papae, 82).

Datierung: Ca. 1297, da der Inhalt sich auf die erste Phase des Streits zwischen Philipp dem Schönen von Frankreich und Bonifaz VIII. bezieht (1296-1297). Allerdings hält Scholz die Jahre 1298 oder 1300 wegen der Anspielungen auf den Streit zwischen Flandern und Frankreich noch für möglich (Scholz, 343-344).

Inhalt: Ein Priester und ein Ritter diskutieren die Oberherrschaft des Papstes über den König. Der Priester formuliert knapp die Argumente für den Papst, der Ritter reagiert darauf mit ausgedehnten Reden und zahlreichen Bibelzitaten. Der Ritter argumentiert, dass der Papst keine weltliche Gewalt ausüben dürfe, da Petrus vor der Wiederauferstehung Jesu als Vikar eingesetzt sei und nur die Macht, die Jesus auf Erden hatte, erben könne. Dann meint der Priester, Unrecht sei Sünde und der Papst könne über Sünde befinden. Als Reaktion darauf unterscheidet der Ritter zwischen Sünde und Unrecht. Der Priester argumentiert, dass die weltliche Macht der geistlichen unterstellt sein soll. Der Ritter gibt nur zu, dass die weltlichen Herrscher für den Unterhalt der Geistlichen aufkommen müssten, allerdings behalten die Schenker das Recht, den Gebrauch der geschenkten Güter zu überprüfen. Der König 
dürfe Steuer von der Kirche verlangen, da er die Kirche (und die Laien seien ja ein Teil davon) schütze. Der letzte angesprochene Punkt ist, ob der Kaiser Hoheit über den König habe, was der Ritter verneint, da der König kaiserliche Gewalt in seinem Königreich ausüben dürfe. Das Gespräch endet abrupt mit der letzten Rede des Ritters.

\section{Editionen:}

Disputatio super potestate praelatis Ecclesiae atque Principibus terrarum commissa, in: Goldast von Heiminsfeld, Melchior, Monarchia sancti Romani Imperii Bd. 1, Frankfurt a. M. 1611 (ND Graz 1960), 13-18.

Sichardius, Simon, Sylloge historico-politico-ecclesiastica, hoc est variorum auctorum superioribus seculis aliquot qui vixerunt, de discrimine potestatis imperialis et ecclesiasticae, imperialisque praeeminenti jurisdictione et auctoritate de imperii Romani ad Germanos translatione de juribus regni et imperii, de commentitia Constantini donatione $E^{2}$. tractatus aliquot... Straßburg 1618, 75-80.

Erickson, Norma N., „A dispute between a Priest and a Knight", Proceedings of the American Philosophical Society 111, 5 (1967), 288-309. Kritischer Text auf der Grundlage von elf Handschriften, gefolgt von einer englischen Übersetzung.

Dyson, Robert W., Three Royalist Tracts 1296-1302: Antequam essent clerici; Disputatio inter Clericum et militem; Quaestio in utramquam partem, Bristol 1999, zu diesem Text xvii-xxvii, Edition 12-45. Kritischer Text auf der Basis derselben Handschriften wie Ericksons Edition, zu der kaum Unterschiede bestehen, neue englische Übersetzung.

\section{Übersetzungen / Bearbeitungen:}

Zweite Hälfte des 14. Jhs. ins Englische, von John Trevisa:

Perry, Aaron J., Dialogus inter militem et clericum, Richard Fitz Ralph's Sermon, Defensio curatorum and Methodius, pe Bygynnyng of pe World and pe Ende of Worldes by John Trevisa, London 1925 (ND Millwood, NY 1987).

Fowler, Trevisa, 145-163.

Somerset, Fiona, Clerical Discourse and Lay Audience in Late Medieval England, Cambridge 1998, 78-93.

Ins Deutsche von Joseph Pollinger (16. Jh.?). Druck:

Ain schöner Dialogus zwischen ainem Priester und Ritter von ainer steür über die gaistlichenn etwan in Franckreich angelegt gehalten, [Augsburg: Sigmund Grimm, um 1523]. VD 16 D 1353.

Große Teile des Dialogs wurden in das Somnium Viridarii eingearbeitet (siehe R64).

Somnium viridarii, Ed. Schnerb-Lièvre, Bd. 2, 529.

\section{Literatur:}

Fowler, David C., The Life and Times of Fohn Trevisa, Medieval Scholar, SeattleLondon 1995.

Miethke, Jürgen, De potestate papae. Die päpstliche Amtskompetenz im Widerstreit der politischen Theorie von Thomas von Aquin bis Wilhelm von Ockham, Tübingen 2000, hier 79-82.

Renna, Thomas J., „Kingship in the Disputatio inter clericum et militem“, Speculum 48 (1973), 675-693. 
Scholz, Richard, Die Publizistik zur Zeit Philipps des Schönen und Bonifaz VIII., Stuttgart 1903, 333-352.

Schmitz, Wolfgang, „Die Kölner Ausgaben des Dialogus inter clericum et militem in buchgeschichtlicher Sicht", Gutenberg-Jahrbuch 74 (1996), 106-112.

Walther, Hans, Das Streitgedicht in der lateinischen Literatur des Mittelalters, München 1920, ND Hildesheim (Georg Olms Verlag) 1984, mit einem Vorwort, Nachträgen und Registern von Paul Gerhard Schmidt, 170-172.

\section{Überlieferung:}

Handschriften:

Aus Ed. Erickson (290-291), alle aus der ersten Hälfte des 15. Jhs.:

Bordeaux, BM, 406, fol. 76r-76v (Fragment).

Cambridge, Corpus Christi College, 156, 22.

Cambridge, St. John's College, 115, 4 (E 12).

Cambridge, St. John's College, 160, 1.

London, BL, Cotton Nero D. VIII, 10, fol. 183-186.

London, BL, Royal 6. E. III, 41, fol. 130-132.

Paris, BnF, lat. 4364, fol. 45v-46r (Fragment).

Paris, BnF, lat. 12464 (St. Germain 394), fol. 158r-158v (Fragment).

Paris, BnF, lat. 15004 (St. Victor 895), fol. 5r-11v.

Vaticano, BAV, Borgh. 29, fol. 24v-29v.

Vaticano, BAV, Vat. lat. 13680, fol. 104r (Fragment).

David C. Fowler fand ferner (Trevisa, 153):

Oxford, BodL, Rawlinson G. 40., hier 15. Jh., fol. 32-38.

Aus Miethke, De potestate (306-307):

Basel, UB, E. I. 11, 15. Jh., fol. 5r-10r.

Gießen, UB, 733, a.1453/54, fol. 107ra-111ra.

München, BSB, Clm 21059, 15. Jh., fol. 41ra-45rb.

Philadelphia, Temple University, Cochran 501, 15. Jh. (2. Viertel), fol. $43 \mathrm{v}-50 \mathrm{v}$.

Praha, KMK, J 30 (Podlaha 1134), fol. 265v-270r.

Praha, NK, VIII. F. 131 (Truhláŕ 567), a. 1445-1481, fol. 230r-236r.

Vaticano, BAV, Reg. lat. 1059, 14. Jh., fol. 165rb-167v.

Vaticano, BAV, Vat. lat. 4100, a. 1429, fol. 1r-7r.

Venezia, BNM, Lat. IV. 20 (= 2372) (Valentinelli 2, 315), 15. Jh., fol. $2 \mathrm{r}-\mathrm{v}$.

Wrocław, BUn, Rehdiger 130, Jh.?, fol.?

Frühdrucke:

Zu den Kölner Drucken vgl. Schmitz.

Laut Angabe in GW haben alle Drucke als Anhang ein Compendium de vita Antichristi. Drucke nicht eingesehen.

Disputacio inter clericum et militem super potestate prelatis ecclesie atque principibus terrarum commissa sub forma dyalogi, [Köln: Drucker von Augustinus, de fide, um 1473]. GW 8261.

Disputacio inter clericum et militem super potestate prelatis ecclesie atque principibus terrarum commissa sub forma dyalogi, [Köln: Nikolaus Götz?, um 1475]. GW 8262. 